\title{
Therapeutic Index Associated with Lymphadenectomy Among Patients with Intrahepatic Cholangiocarcinoma: Which Patients Benefit the Most from Nodal Evaluation?
}

\author{
Kota Sahara, $\mathrm{MD}^{1,2}$, Diamantis I. Tsilimigras, $\mathrm{MD}^{\mathbf{1}}$, Katiuscha Merath, $\mathrm{MD}^{\mathbf{1}}$, Fabio Bagante, $\mathrm{MD}^{\mathbf{3}}$, \\ Alfredo Guglielmi, $\mathrm{MD}^{3}$, Luca Aldrighetti, $\mathrm{MD}^{4}$, Matthew Weiss, $\mathrm{MD}^{5}$, Todd W. Bauer, $\mathrm{MD}^{6}$, \\ Sorin Alexandrescu, $\mathrm{MD}^{7}$, George A. Poultsides, $\mathrm{MD}^{8}$, Shishir K. Maithel, MD ${ }^{9}$, Hugo P. Marques, $\mathrm{MD}^{10}$, \\ Guillaume Martel, $\mathrm{MD}^{11}$, Carlo Pulitano, $\mathrm{MD}^{12}$, Feng Shen, $\mathrm{MD}^{13}$, Olivier Soubrane, $\mathrm{MD}^{14}$, \\ B. Groot Koerkamp, $\mathrm{MD}^{15}$, Ryusei Matsuyama, $\mathrm{MD}, \mathrm{PhD}^{2}$, Itaru Endo, $\mathrm{MD}$, $\mathrm{PhD}^{2}$, and \\ Timothy M. Pawlik, MD, MPH, PhD, FACS ${ }^{1,16}$ (])
}

${ }^{1}$ Department of Surgery, Division of Surgical Oncology, The Ohio State University Wexner Medical Center and James Comprehensive Cancer Center, Columbus, OH; ${ }^{2}$ Department of Gastroenterological Surgery, Yokohama City University School of Medicine, Yokohama, Japan; ${ }^{3}$ Department of Surgery, University of Verona, Verona, Italy; ${ }^{4}$ Department of Surgery, Ospedale San Raffaele, Milan, Italy; ${ }^{5}$ Department of Surgery, Johns Hopkins Hospital, Baltimore, MD; ${ }^{6}$ Department of Surgery, University of Virginia, Charlottesville, VA; ${ }^{7}$ Department of Surgery, Fundeni Clinical Institute, Bucharest, Romania; ${ }^{8}$ Department of Surgery, Stanford University, Stanford, CA; ${ }^{9}$ Department of Surgery, Emory University, Atlanta, GA; ${ }^{10}$ Department of Surgery, Curry Cabral Hospital, Lisbon, Portugal; ${ }^{11}$ Department of Surgery, University of Ottawa, Ottawa, ON, Canada; ${ }^{12}$ Department of Surgery, Royal Prince Alfred Hospital, University of Sydney, Sydney, NSW, Australia; ${ }^{13}$ Department of Surgery, Eastern Hepatobiliary Surgery Hospital, Shanghai, China;

${ }^{14}$ Department of Hepatobiliopancreatic Surgery and Liver Transplantation, AP-HP, Beaujon Hospital, Clichy, France;

${ }^{15}$ Department of Surgery, Erasmus University Medical Centre, Rotterdam, The Netherlands; ${ }^{16}$ Department of Surgery, The Urban Meyer III and Shelley Meyer Chair for Cancer Research, Wexner Medical Center, The Ohio State University, Columbus, $\mathrm{OH}$

\section{ABSTRACT}

Background. Although lymph node metastasis (LNM) is an important prognostic indicator for patients with intrahepatic cholangiocarcinoma (ICC), the benefit and indication for lymphadenectomy remain unclear.

Methods. Patients diagnosed with ICC between 1990 and 2016 were identified in the international multi-institutional

Kota Sahara and Diamantis Tsilimigras contributed equally to this work.

Electronic supplementary material The online version of this article (https://doi.org/10.1245/s10434-019-07483-9) contains supplementary material, which is available to authorized users.

(C) Society of Surgical Oncology 2019

First Received: 15 February 2019;

Published Online: 31 May 2019

T. M. Pawlik, MD, MPH, PhD, FACS

e-mail: Tim.Pawlik@osumc.edu dataset. To determine the survival benefit from lymphadenectomy, the therapeutic index was calculated by multiplying the frequency of LNM in a particular group of patients by the 3-year cancer-specific survival (CSS) rate of patients with LNM in that subgroup.

Results. Among 471 patients who met the inclusion criteria, approximately half had LNM ( $n=205,43.5 \%)$. The median number of resected and metastatic LNs were 4 [interquartile range (IQR) 2-8] and 0 (IQR 0-1), respectively. Three-year CSS in the entire cohort was $29.9 \%$, reflecting a therapeutic index value of 13.0. The therapeutic index was lower among patients with major vascular invasion (5.4), preoperative carcinoembryonic antigen $($ CEA) $>5.0$ (8.2), and LNM in areas other than the hepatoduodenal ligament (5.2). Of note, a therapeutic index difference of more than 10 points was noted only when examining the number of LNs harvested [1-2 (4.1) vs. 3-6 (16.1) vs. $\geq 7$ (17.8)].

Conclusion. The survival benefit derived from lymphadenectomy was poor among patients with major 
vascular invasion, CEA $>5.0$, and LNM in areas other than the hepatoduodenal ligament. Resection of three or more LNs was associated with the highest therapeutic value among patients with LNM.

Intrahepatic cholangiocarcinoma (ICC) is a relatively rare cancer with an increasing incidence in the US over the last three decades. ${ }^{1,2}$ Despite the unfavorable outcomes of patients diagnosed with ICC, surgery remains the mainstay of potentially curative treatment for patients with ICC amenable to resection. ${ }^{3-7}$ Appropriate oncologic principles of ICC resection include both complete removal of the tumor as well as a lymphadenectomy. While lymphadenectomy is essential to adequately stage the disease and decrease the risk of locoregional recurrence, ${ }^{8,9}$ the extent of lymphadenectomy and its related therapeutic benefit remain a topic of debate. ${ }^{6,10-15}$ Indeed, an analysis of the Surveillance, Epidemiology, and End Results (SEER) database noted that only one-half of patients had pathological examination of at least one regional lymph node (LN). ${ }^{10,11}$ To this end, data are needed to define the potential therapeutic benefit, as well as identify the group of patients who might benefit the most from LN dissection (LND), to guide treatment planning and improve long-term outcomes. ${ }^{16}$

First proposed by Sasako et al., the therapeutic index is a simple metric that aims to determine the survival benefit derived from LND among patients undergoing surgery for gastrointestinal (GI) malignancies. ${ }^{17}$ The rationale of this metric is that LND should ideally be performed among patients with a high predicted incidence of LN metastasis and who could, therefore, derive a survival benefit. ${ }^{17}$ Although first applied to evaluate the survival benefit of LND among patients with gastric cancer, ${ }^{16,18,19}$ the concept of nodal therapeutic index has also been applied to patients with colorectal cancer. ${ }^{20}$ In fact, the therapeutic index has been included in the Japanese Classification of Gastric Carcinoma (JCGC), suggesting wide acceptance among certain clinicians. ${ }^{21}$ The concept of $\mathrm{LN}$ therapeutic index has not been investigated, applied, or validated among patients with hepatobiliary malignancies. As such, the objective of the current study was to examine the survival benefit of lymphadenectomy among patients undergoing resection for ICC utilizing the therapeutic index. Furthermore, we sought to identify the subgroup of patients most likely to have derived the least versus most benefit from LND at the time of surgery for ICC.

\section{METHODS}

\section{Study Population and Data Collection}

Patients undergoing surgical resection for ICC between January 1990 and December 2016 were identified in a multi-institutional database incorporating data from 15 tertiary institutions. For the purpose of this study, only patients with histologically confirmed ICC who underwent curative intent liver resection were analyzed. Patients who did not undergo lymphadenectomy were excluded from the analyses, as well as patients with macroscopic positive surgical margins (R2 resection), 30-day mortality after surgery, and missing follow-up data. The Institutional Review Boards of the participating institutions approved this study.

Patient demographic and clinicopathologic data were extracted, including age, sex, race, American Society of Anesthesiologists (ASA) class, the presence of cirrhosis, preoperative serum level of carbohydrate antigen (CA) 19-9 and carcinoembryonic antigen (CEA), type of resection, number of ICCs, tumor size, lymph nodal status, location of LN metastasis (LNM), morphological type (MF: mass-forming; IG: intraductal growth; PI: periductal infiltrating), tumor grade and margin status, major or minor vascular invasion, and the presence of perioperative chemotherapy. Major hepatectomy was defined as the resection of three or more Couinaud segments. ${ }^{22} \mathrm{LN}$ basins were classified into two categories: hepatoduodenal ligament, and other station including celiac and para-aortic LNs. Major vascular invasion was defined as invasion of the first- and second-order branches of the portal vein or hepatic arteries, or as invasion of one or more of the three hepatic veins. In contrast, microvascular invasion was defined as intraparenchymal vascular involvement identified on histological examination. ${ }^{23}$

\section{Therapeutic Index}

The therapeutic index of lymphadenectomy was calculated by multiplying the frequency of LNM in a particular group of patients by the 3-year cancer-specific survival (CSS) rate of patients with LNM in that subgroup, as previously reported. ${ }^{18}$ The therapeutic index was estimated for both clinicopathological and LN-related characteristics, as previously described. ${ }^{18}$ The incidence of LNM was calculated by dividing the number of patients with metastatic LN by the total number of patients in this particular subgroup. The 3-year CSS was calculated for each group of patients with metastatic LNs, irrespective of the metastasis to LN stations or number of LNs harvested. In cases where a therapeutic index interval, defined as the difference of the 
therapeutic index values between two groups, was more than 10 , LND was considered meaningful. ${ }^{16,19}$

\section{Statistical Analysis}

Descriptive statistics were presented as median [interquartile range (IQR)] and frequency (\%) for continuous and categorical variables, respectively. Statistical significance was assessed at $\alpha=0.05$. A logistic multivariable regression model was used to identify independent predictors of LNM. Patients who were alive or had died of a cause other than ICC were censored for analysis of CSS. CSS was defined as the time from the date of first liver resection to the date of death or last follow-up. CSS was calculated by creating Kaplan-Meier curves, and survival was compared using log-rank tests. The association of clinicopathologic variables with CSS was evaluated by using a Cox proportional hazards model. The multivariable model was developed by fitting bivariate generalized estimating equation (GEE) models for each confounder and including the variable in the subsequent stage of analysis if the statistical significance was $p<0.05$ for the primary outcome. ${ }^{24}$ All statistical analyses were performed using SPSS version 25 (IBM Corporation, Armonk, NY, USA).

\section{RESULTS}

\section{Characteristics of Patients Undergoing \\ Lymphadenectomy}

Among 1086 patients, 648 (59.6\%) patients were treated in Western hospitals, whereas $438(40.4 \%)$ were treated in Eastern hospitals (electronic supplementary Table S1). In total, 522 patients $(48.1 \%)$ underwent LND, whereas 564 patients $(51.9 \%$ ) did not (electronic supplementary Fig. S1). Among the 522 patients who underwent LND, 51 were excluded due to missing data on LN status and survival, therefore 471 patients remained in the final analytic cohort. Median patient age at the time of surgery was 62 years (IQR 53-70) and approximately one-half of patients were male $(n=220,46.7 \%)$. The majority of patients were Caucasian $(n=297,66.3 \%)$, had an ASA score $\leq 2(n=256,54.4 \%)$, and did not have cirrhosis $(n=380,80.7 \%)$. The preoperative median CA19-9 and CEA were $97 \mathrm{UI} / \mathrm{mL}$ (IQR 31-391) and $2.4 \mathrm{ng} / \mathrm{mL}$ (IQR 1.4-4.5), respectively; only a minority of patients received neoadjuvant chemotherapy ( $n=57,13.6 \%)$. Most patients underwent a minor hepatectomy $(n=387,82.7 \%)$, whereas a smaller subset underwent a major hepatectomy $(n=81,17.3 \%)$. Most patients had unifocal disease $(n=345,78.4 \%)$ and the median tumor size was $6.7 \mathrm{~cm}$ (IQR 4.5-9.0). Of note, approximately half of the patients had LNM ( $n=205,43.5 \%)$. Perhaps not surprisingly, the more common area of LNM was the hepatoduodenal ligament $(n=373,79.7 \%)$. The median number of LNs harvested and metastatic LNs was 4 (IQR 2-8) and 0 (IQR $0-1)$, respectively. The majority of patients had an R0 resection $(n=379,80.5 \%)$, MF or IG morphologic ICC type $(n=334,76.3 \%)$, and well- to moderate-grade tumors $(n=342,74.2 \%)$, whereas the minority had major vascular invasion $(n=78,16.6 \%)$. Microvascular invasion was present in $42.2 \%(n=198)$ of resected ICCs. Approximately half of the patients received adjuvant chemotherapy $(n=228,51.5 \%)$ (Table 1).

\section{Clinicopathological Factors Associated with Lymph Node Dissection, Lymph Node Metastasis, and Cancer- Specific Survival}

While the geographical region of treating centers was not associated with the use of LND, patients treated more recently [2013-2016; odds ratio (OR) 2.46, 95\% confidence interval (CI) 1.25-4.88], as well as individuals with multifocal disease (OR 2.06, 95\% CI 1.59-4.27), tumor $>5 \mathrm{~cm}$ (OR 1.73, 95\% CI 1.08-2.78), PI or $\mathrm{MF}+\mathrm{PI}$ morphologic type (OR 20.0, 95\% CI 4.15-96.7), and microvascular invasion (OR 1.98, 95\% CI 1.14-3.43) were more likely to undergo LND (electronic supplementary Table S2). Among patients with LND, preoperative CEA $>5.0$ (OR 2.21, 95\% CI 1.20-4.08), PI or MF + PI morphologic type (OR 1.75, 95\% CI 1.12-2.72), and major vascular invasion remained associated with LNM (OR $1.76,95 \%$ CI 1.08-2.88) on bivariate analysis (Table 2).

In analyzing CSS, the bivariate analysis revealed several factors that were associated with poorer CSS, including preoperative CA19-9 > 37 [hazard ratio (HR) 1.54, 95\% CI 1.06-2.23], preoperative CEA $>5.0$ (HR 1.43, 95\% CI 1.05-1.96), multifocal disease (HR 1.79, 95\% CI $1.31-2.44$ ), tumor size $>5.0 \mathrm{~cm}$ (HR $1.43,95 \%$ CI 1.05-1.96), LNM (HR 3.01, 95\% CI 2.25-4.01), poor/undifferentiated grade (HR 1.67, 95\% CI 1.23-2.26), and major vascular invasion (HR 1.65, 95\% CI 1.17-2.34). On multivariable analysis, preoperative CEA $>5.0$ (HR 2.65, 95\% CI 1.60-4.38), tumor size $>5.0 \mathrm{~cm}$ (HR 1.96, 95\% CI 1.19-3.21), and LNM (HR 2.74, 95\% CI 1.69-4.43) remained associated with CSS (Table 3).

\section{Survival and Therapeutic Index by Perioperative Factors}

The median follow-up was 17.9 months (IQR 8.7-36.5). Overall, the frequency of LNM was $43.5 \%$, and 3-year CSS in the entire cohort was $29.9 \%$ (Fig. 1a) for a therapeutic index value of $13(0.435 \times 29.9)$. Irrespective of other factors, patients with major vascular invasion had the worst 
TABLE 1 Demographic and patient characteristics in the entire cohort

\begin{tabular}{|c|c|}
\hline Variable & $N(\%)$ \\
\hline Age, years [median (IQR)] & $62(53-70)$ \\
\hline Male & $220(46.7)$ \\
\hline \multicolumn{2}{|l|}{ Race } \\
\hline Caucasian & $297(66.3)$ \\
\hline African American & $23(5.1)$ \\
\hline Asian & $115(25.7)$ \\
\hline Other & $13(2.9)$ \\
\hline \multicolumn{2}{|l|}{ ASA score } \\
\hline$\leq 2$ & $256(54.4)$ \\
\hline$>2$ & $215(45.6)$ \\
\hline Cirrhosis & $17(4.3)$ \\
\hline CA19-9, UI/mL [median (IQR)] & $97(31-391)$ \\
\hline CEA, ng/mL [median (IQR)] & $2.4(1.4-4.5)$ \\
\hline \multicolumn{2}{|l|}{ Neoadjuvant therapy } \\
\hline No & $362(86.4)$ \\
\hline Yes & $57(13.6)$ \\
\hline \multicolumn{2}{|l|}{ Type of resection } \\
\hline Minor & $81(17.3)$ \\
\hline Major & $387(82.7)$ \\
\hline \multicolumn{2}{|l|}{ Location } \\
\hline Unifocal & $345(78.4)$ \\
\hline Multifocal & $95(21.6)$ \\
\hline Tumor size, cm [median (IQR)] & $6.7(4.5-9.0)$ \\
\hline \multicolumn{2}{|l|}{ Lymph node metastasis } \\
\hline No & $266(56.5)$ \\
\hline Yes & $205(43.5)$ \\
\hline \multicolumn{2}{|l|}{ Lymph node area } \\
\hline Hepatoduodenal ligament & 373 (79.7) \\
\hline Other station & $95(20.3)$ \\
\hline No. of lymph nodes harvested [median (IQR)] & $4(2-8)$ \\
\hline \multicolumn{2}{|l|}{ No. of lymph nodes harvested } \\
\hline $1-2$ & $147(33.7)$ \\
\hline $3-6$ & $148(33.9)$ \\
\hline$\geq 7$ & $141(32.3)$ \\
\hline No. of lymph node metastases [median (IQR)] & $0(0-1)$ \\
\hline \multicolumn{2}{|l|}{ Margin status } \\
\hline R0 & $379(80.5)$ \\
\hline R1 & $92(19.5)$ \\
\hline \multicolumn{2}{|l|}{ Morphologic type } \\
\hline MF, IG & $334(76.3)$ \\
\hline $\mathrm{PI}, \mathrm{MF}+\mathrm{PI}$ & $104(23.7)$ \\
\hline \multicolumn{2}{|l|}{ Grade } \\
\hline Well to moderate & $342(74.2)$ \\
\hline Poor to undifferentiated & $119(25.8)$ \\
\hline \multicolumn{2}{|l|}{ Major vascular invasion } \\
\hline No & $78(16.6)$ \\
\hline Yes & $392(83.4)$ \\
\hline
\end{tabular}

TABLE 1 continued

\begin{tabular}{ll}
\hline Variable & $N(\%)$ \\
\hline Microvascular invasion & \\
No & $198(42.2)$ \\
Yes & $271(57.8)$ \\
Adjuvant chemotherapy & \\
No & $215(48.5)$ \\
Yes & $228(51.5)$ \\
\hline
\end{tabular}

Data are expressed as $n(\%)$ unless otherwise specified

$I Q R$ interquartile range, ASA American Society of Anesthesiologists, $C A$ carbohydrate antigen, $C E A$ carcinoembryonic antigen, $M F$ massforming, $I G$ intraductal growth, $P I$ periductal infiltrating

3-year CSS (9.8\%), followed by patients with one to two LNs harvested (10.8\%), CEA > $5 \mathrm{ng} / \mathrm{mL}(13.7 \%)$, and metastatic LNs in areas other than the hepatoduodenal ligament (15.0\%). The highest therapeutic value was noted among patients with seven or more LNs resected (17.8), followed by CEA $<5 \mathrm{ng} / \mathrm{mL}$ (16.8), and no major vascular invasion (15). Interestingly, an index difference of more than 10 points was noted only when examining the number of LNs harvested [1-2 (4.1) vs. 3-6 (16.1) vs. $\geq 7$ (17.8)]. In turn, patients with three or more LNs harvested had an increased OS compared with patients with one to two LNs resected when the LNs were positive $(p=0.04)$, whereas no survival benefit was noted among patients with nonmetastatic LNs $(p=0.41)$ (Fig. 1b, c). Large index differences, although not reaching a value of 10 , were also noted for no major vascular invasion [index difference: 9.6; no (15.0) vs. yes (5.4)], CEA $\leq 5 \mathrm{ng} / \mathrm{mL}[8.4$; CEA $\leq 5.0$ (16.8) vs. $>5.0(8.2)]$, and LNM at the hepatoduodenal ligament [7.3; hepatoduodenal ligament (12.5) vs. other station (5.2)] (Table 4).

\section{DISCUSSION}

Lymphadenectomy is an important part of the oncologic resection of ICC as it aids in the adequate staging of the disease, as well as likely decreases locoregional recurrence. $^{8,9}$ Indeed, LND has been suggested to be the standard of care in the treatment of ICC, as reflected in the 8th edition of the American Joint Committee on Cancer (AJCC) staging manual, which suggests at least six LNs be examined to appropriately stage the disease. ${ }^{23}$ While resection is the cornerstone of potentially curative therapy for ICC, the prognosis of patients with ICC still remains dismal. In addition, the role of LND has been debated, with clinicians questioning the therapeutic benefit of an LND in the context of ICC treatment. Interestingly, previous nationwide surveys have revealed a relatively low implementation of lymphadenectomy in the surgical treatment of 
TABLE 2 Logistic regression analysis of clinicopathological factors associated with lymph node metastasis

\begin{tabular}{|c|c|c|c|c|}
\hline \multirow[t]{2}{*}{ Variable } & \multicolumn{2}{|c|}{ Bivariate analysis } & \multicolumn{2}{|c|}{ Multivariable analysis } \\
\hline & OR & $95 \% \mathrm{CI}$ & OR & $95 \% \mathrm{CI}$ \\
\hline \multicolumn{5}{|l|}{ Age, years } \\
\hline$\leq 65$ & Ref & & & \\
\hline$>65$ & 0.78 & $0.53-1.15$ & & \\
\hline \multicolumn{5}{|l|}{ Sex } \\
\hline Male & Ref & & & \\
\hline Female & 0.78 & $0.54-1.12$ & & \\
\hline \multicolumn{5}{|l|}{ Year } \\
\hline 1990-2008 & Ref & & Ref & \\
\hline 2009-2012 & 1.77 & $1.15-2.73$ & 1.91 & $0.92-4.00$ \\
\hline 2013-2016 & 1.61 & $1.00-2.59$ & 1.34 & $0.64-2.82$ \\
\hline \multicolumn{5}{|l|}{ Hospital } \\
\hline East & Ref & & Ref & \\
\hline West & 0.27 & $0.17-0.41$ & 0.26 & $0.15-0.47$ \\
\hline \multicolumn{5}{|l|}{ CA19-9, UI/mL } \\
\hline$\leq 37$ & Ref & & & \\
\hline$>37$ & 1.40 & $0.86-2.27$ & & \\
\hline \multicolumn{5}{|l|}{ CEA, ng/mL } \\
\hline$\leq 5.0$ & Ref & & Ref & \\
\hline$>5.0$ & 2.21 & $1.20-4.08$ & 1.91 & $0.96-3.78$ \\
\hline \multicolumn{5}{|l|}{ Location } \\
\hline Unifocal & Ref & & & \\
\hline Multifocal & 1.29 & $0.82-2.01$ & & \\
\hline \multicolumn{5}{|l|}{ Tumor size, $\mathrm{cm}$} \\
\hline$\leq 5.0$ & Ref & & & \\
\hline$>5.0$ & 0.98 & $0.66-1.44$ & & \\
\hline \multicolumn{5}{|l|}{ Morphologic type } \\
\hline MF, IG & Ref & & Ref & \\
\hline $\mathrm{PI}, \mathrm{MF}+\mathrm{PI}$ & 1.75 & $1.12-2.72$ & 1.52 & $0.74-3.10$ \\
\hline \multicolumn{5}{|l|}{ Grade } \\
\hline Well to moderate & Ref & & & \\
\hline Poor to undifferentiated & 1.08 & $0.71-1.64$ & & \\
\hline \multicolumn{5}{|l|}{ Major vascular invasion } \\
\hline No & Ref & & Ref & \\
\hline Yes & 1.76 & $1.08-2.88$ & 1.33 & $0.61-2.91$ \\
\hline \multicolumn{5}{|l|}{ Microvascular invasion } \\
\hline No & Ref & & & \\
\hline Yes & 1.15 & $0.79-1.66$ & & \\
\hline
\end{tabular}

$O R$ odds ratio, $C I$ confidence interval, $C A$ carbohydrate antigen, $C E A$ carcinoembryonic antigen, $M F$ mass-forming, $I G$ intraductal growth, $P I$ periductal infiltrating

ICC, which has not changed over the last decade across the US. $^{25,26}$ The current study was important because we identified a group of patients who seemingly derived the most benefit (so-called 'therapeutic value') from LND by utilizing a previously described metric-the therapeutic index. ${ }^{17}$ Of note, the highest therapeutic value of LND was noted among patients with resection of seven or more LNs (17.8), as well as CEA $\leq 5 \mathrm{ng} / \mathrm{mL}$ (16.8), tumor size $\leq 5.0 \mathrm{~cm}$ (15.6), and no major vascular invasion (15.0). Perhaps of more interest, a therapeutic index interval of more than 10 was noted only among patients having three or more LNs resected, compared with resection of one to 
TABLE 3 Cox regression analysis of clinicopathological factors associated with cancer-specific survival

\begin{tabular}{|c|c|c|c|c|}
\hline \multirow[t]{2}{*}{ Variable } & \multicolumn{2}{|c|}{ Bivariate analysis } & \multicolumn{2}{|c|}{ Multivariable analysis } \\
\hline & OR & $95 \% \mathrm{CI}$ & OR & $95 \% \mathrm{CI}$ \\
\hline \multicolumn{5}{|l|}{ Age, years } \\
\hline$\leq 65$ & Ref & & & \\
\hline$>65$ & 0.76 & $0.56-1.04$ & & \\
\hline \multicolumn{5}{|l|}{ Sex } \\
\hline Male & Ref & & & \\
\hline Female & 1.30 & $0.98-1.72$ & & \\
\hline \multicolumn{5}{|l|}{ Year } \\
\hline 1990-2008 & Ref & & & \\
\hline 2009-2012 & 1.17 & $0.86-1.59$ & & \\
\hline 2013-2016 & 0.91 & $0.55-1.50$ & & \\
\hline \multicolumn{5}{|l|}{ Hospital } \\
\hline East & Ref & & Ref & \\
\hline West & 0.71 & $0.53-0.96$ & 0.82 & $0.50-1.34$ \\
\hline \multicolumn{5}{|l|}{ CA19-9, UI/mL } \\
\hline$\leq 37$ & Ref & & Ref & \\
\hline$>37$ & 1.54 & $1.06-2.23$ & 0.91 & $0.58-1.45$ \\
\hline \multicolumn{5}{|l|}{ CEA, ng/mL } \\
\hline$\leq 5.0$ & Ref & & Ref & \\
\hline$>5.0$ & 2.81 & $1.85-4.28$ & 2.65 & $1.60-4.38$ \\
\hline \multicolumn{5}{|l|}{ Neoadjuvant therapy } \\
\hline No & Ref & & & \\
\hline Yes & 1.12 & $0.72-1.74$ & & \\
\hline \multicolumn{5}{|l|}{ Location } \\
\hline Unifocal & Ref & & Ref & \\
\hline Multifocal & 1.79 & $1.31-2.44$ & 1.31 & $0.75-2.29$ \\
\hline \multicolumn{5}{|l|}{ Tumor size, $\mathrm{cm}$} \\
\hline$\leq 5.0$ & Ref & & Ref & \\
\hline$>5.0$ & 1.43 & $1.05-1.96$ & 1.96 & $1.19-3.21$ \\
\hline \multicolumn{5}{|l|}{ Type of resection } \\
\hline Minor resection & Ref & & & \\
\hline Major resection & 1.09 & $0.76-1.57$ & & \\
\hline \multicolumn{5}{|l|}{ Lymph node metastasis } \\
\hline No & Ref & & Ref & \\
\hline Yes & 3.01 & $2.25-4.01$ & 2.74 & $1.69-4.43$ \\
\hline \multicolumn{5}{|l|}{ Margin status } \\
\hline R0 & Ref & & & \\
\hline $\mathrm{R} 1$ & 1.24 & $0.87-1.78$ & & \\
\hline \multicolumn{5}{|l|}{ Morphologic type } \\
\hline MF, IG & Ref & & & \\
\hline $\mathrm{PI}, \mathrm{MF}+\mathrm{PI}$ & 1.34 & $0.98-1.84$ & & \\
\hline \multicolumn{5}{|l|}{ Grade } \\
\hline Well to moderate & Ref & & Ref & \\
\hline Poor to undifferentiated & 1.67 & $1.23-2.26$ & 1.20 & $0.70-2.06$ \\
\hline \multicolumn{5}{|l|}{ Major vascular invasion } \\
\hline No & Ref & & Ref & \\
\hline Yes & 1.65 & $1.17-2.34$ & 1.44 & $0.84-2.47$ \\
\hline
\end{tabular}


TABLE 3 continued

\begin{tabular}{|c|c|c|c|c|}
\hline \multirow[t]{2}{*}{ Variable } & \multicolumn{2}{|c|}{ Bivariate analysis } & \multicolumn{2}{|c|}{ Multivariable analysis } \\
\hline & OR & $95 \% \mathrm{CI}$ & OR & $95 \% \mathrm{CI}$ \\
\hline \multicolumn{5}{|c|}{ Microvascular invasion } \\
\hline No & Ref & & & \\
\hline Yes & 1.00 & $0.75-1.33$ & & \\
\hline \multicolumn{5}{|c|}{ Adjuvant chemotherapy } \\
\hline No & Ref & & & \\
\hline Yes & 1.07 & $0.80-1.44$ & & \\
\hline
\end{tabular}

$O R$ odds ratio, $C I$ confidence interval, $C A$ carbohydrate antigen, $C E A$ carcinoembryonic antigen, $M F$ mass-forming, $I G$ intraductal growth, $P I$ periductal infiltrating

(A)

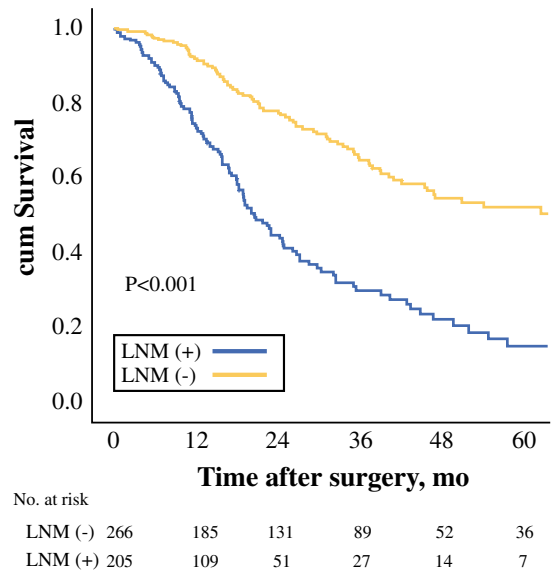

(B)

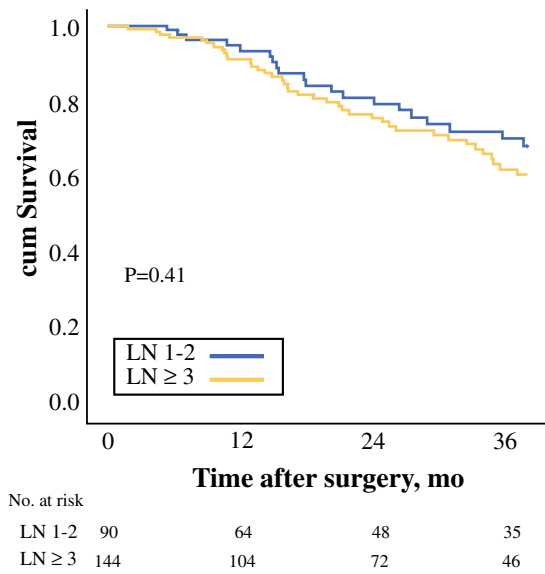

(C)

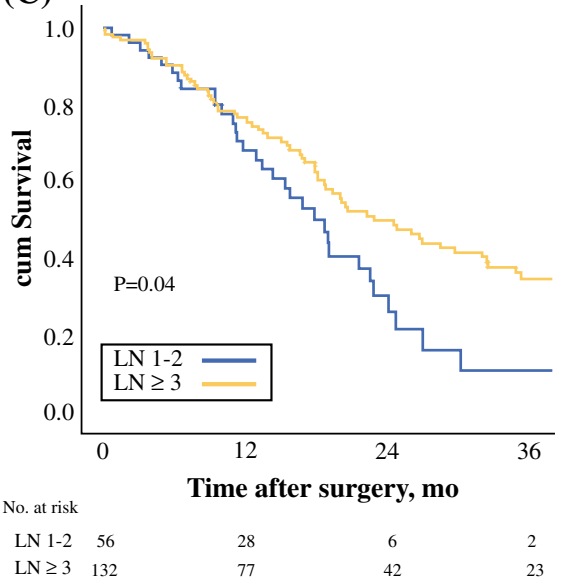

FIG. 1 Kaplan-Meier curves showing cancer-specific survival among patients with LND in total (a), and among patients without (b) and with LNM (c), stratified by the number of LNs harvested. LND lymph node dissection, $L N M$ lymph node metastasis, $L N s$ lymph nodes, Cum cumulative

two LNs [1-2 (4.1) vs. 3-6 (16.1) vs. $\geq 7$ (17.8)]. In addition, a better prognosis was noted among patients with LNM and three or more LNs harvested. In contrast, the lowest therapeutic value of LND was noted among patients with major vascular invasion (index 5.4), one to two LNs resected (4.1), and LN metastases at stations other than the hepatoduodenal ligament (5.2). To the best of our knowledge, this is the first study to utilize the therapeutic index to evaluate the therapeutic benefit associated with LND among patients treated for ICC.

The therapeutic index was first proposed by Sasako et al. in an attempt to evaluate the role of LND in the treatment of gastric cancer. ${ }^{17}$ The therapeutic index metric calculates the therapeutic efficacy of LND among patients with certain characteristics, or LND in certain stations, without taking other nodal status into account, thus avoiding stage migration. ${ }^{17}$ The rationale of the therapeutic index, which is calculated easily using frequency of LNM and long-term survival among patients having LNM, is that LND should ideally be performed among patients with a higher predicted incidence of LNM who could derive a benefit from LND. ${ }^{17}$ Indeed, the optimal number of LNs harvested should be assessed not only on the basis of precise staging related to patients without LNM but also as a means of eliminating locoregional disease among patients with LNM. Given the debated role of LND in the treatment of patients with ICC, ${ }^{20}$ we sought to apply this metric in the evaluation of the therapeutic value of LND among patients undergoing surgery for ICC. Of note, the data demonstrated that resection of three to six LNs and seven or more LNs was associated with a considerable therapeutic index compared with patients who had one or two harvested LNs (with the difference being more than 10), which was in line with the 8th edition of the AJCC staging manual, which recommends six or more LNs be evaluated to stage ICC properly. ${ }^{23}$ 
TABLE 4 Therapeutic index stratified by clinicopathological factors

\begin{tabular}{|c|c|c|c|}
\hline Variable & Frequency of LNM & 3-year CSS (\%) & Therapeutic index \\
\hline Overall & $0.435(205 / 471)$ & 29.9 & 13.0 \\
\hline \multicolumn{4}{|l|}{ CA19-9, UI/mL } \\
\hline$\leq 37$ & $0.406(39 / 96)$ & 35.8 & 14.5 \\
\hline$>37$ & $0.489(110 / 225)$ & 30.4 & 14.9 \\
\hline \multicolumn{4}{|l|}{$\mathrm{CEA}, \mathrm{ng} / \mathrm{mL}$} \\
\hline$\leq 5.0$ & $0.404(76 / 188)$ & 41.7 & 16.8 \\
\hline$>5.0$ & $0.600(33 / 55)$ & 13.7 & 8.2 \\
\hline \multicolumn{4}{|l|}{ Location } \\
\hline Unifocal & $0.418(152 / 364)$ & 32.9 & 13.8 \\
\hline Multifocal & $0.480(47 / 98)$ & 21.2 & 10.2 \\
\hline \multicolumn{4}{|l|}{ Tumor size, $\mathrm{cm}$} \\
\hline$\leq 5.0$ & $0.438(67 / 153)$ & 35.7 & 15.6 \\
\hline$>5.0$ & $0.432(136 / 315)$ & 27.8 & 12.0 \\
\hline \multicolumn{4}{|l|}{ Morphologic type } \\
\hline MF, IG & $0.419(140 / 334)$ & 32.9 & 13.8 \\
\hline $\mathrm{PI}, \mathrm{MF}+\mathrm{PI}$ & $0.558(58 / 104)$ & 25.3 & 14.1 \\
\hline \multicolumn{4}{|l|}{ Grade } \\
\hline Well to moderate & $0.436(149 / 342)$ & 32.7 & 14.3 \\
\hline Poor to undifferentiated & $0.454(54 / 119)$ & 22.6 & 10.3 \\
\hline \multicolumn{4}{|l|}{ Major vascular invasion } \\
\hline No & $0.411(161 / 392)$ & 36.4 & 15.0 \\
\hline Yes & $0.551(43 / 78)$ & 9.8 & 5.4 \\
\hline \multicolumn{4}{|l|}{ Lymph node area } \\
\hline Hepatoduodenal ligament & $0.386(173 / 448)$ & 32.3 & 12.5 \\
\hline Other station & $0.347(33 / 95)$ & 15.0 & 5.2 \\
\hline \multicolumn{4}{|l|}{ No. of LNs harvested } \\
\hline $1-2$ & $0.381(56 / 147)$ & 10.8 & 4.1 \\
\hline $3-6$ & $0.466(69 / 148)$ & 34.5 & 16.1 \\
\hline$\geq 7$ & $0.504(71 / 141)$ & 35.3 & 17.8 \\
\hline
\end{tabular}

$L N M$ lymph node metastasis, $C S S$ cancer-specific survival, $C A$ carbohydrate antigen, $C E A$ carcinoembryonic antigen, $M F$ mass-forming, $I G$ intraductal growth, PI periductal infiltrating, $L N s$ lymph nodes

Previous studies have demonstrated no improvement in survival or recurrence associated with routine lymphadenectomy. ${ }^{7,9,27-30}$ In particular, Hu et al. reported that routine lymphadenectomy may not improve long-term survival among patients with resectable ICC and no evidence of LNM preoperatively. ${ }^{27}$ Of note, lymphadenectomy may even be associated with an increased risk of complications in certain subsets of patients. For example, among patients with cirrhosis, the incidence of complications was $71 \%$ among patients who underwent a lymphadenectomy versus $23 \%$ among patients who did not. ${ }^{31}$ To this end, surgeons need to weigh the risks and potential benefit derived by lymphadenectomy for each patient. In the current study, the survival benefit reflected by the therapeutic index was lower among patients with major vascular invasion (5.4), preoperative
CEA > 5.0 (8.2), and LNM in stations other than hepatoduodenal ligament (5.2), suggesting that these factors may indicate systemic spread rather than locally advanced disease, and thus lymphadenectomy may not be as beneficial to help stage patients or provide a therapeutic benefit. In fact, lymphadenectomy beyond the hepatoduodenal ligament, including the common hepatic artery basin, is unlikely to improve outcomes, even if macroscopic radical LND can be achieved. ${ }^{32}$ Therefore, the adoption of routine lymphadenectomy for patients with major vascular invasion, preoperative CEA $>5.0$, or LNM in extended areas may not be warranted. In contrast, patients who do not have these features should be strongly considered for LND as the therapeutic index associated with this procedure suggests a benefit for this subset of individuals. Hence, future 
prospective studies are needed to verify the therapeutic value of LND among select patients with and without the unfavorable prognostic factors.

While one strength of the current study was that it involved multiple centers, the multi-institutional nature of the study may have possibly introduced certain biases (i.e. selection bias of patients who were offered surgery and lymphadenectomy, missing information on variables of interest, such as CEA, CA19-9 levels, variability in treatment methods, and follow-up schemas among institutions). It was also not possible to standardize the cut-off value of the therapeutic index relative to lymphadenectomy for a certain subgroup; however, using the index, we were able to compare subgroups in regard to the relative therapeutic value of lymphadenectomy. ${ }^{20}$ In addition, analysis of the therapeutic value of LND by ICC 'sidedness' was not feasible in the current study. Finally, information on adjuvant therapy and its potential impact on survival was not included in the analysis of the therapeutic index. Even though data from the BILCAP study have suggested that adjuvant capecitabine improved survival after resection of biliary tract cancers, ${ }^{33}$ the PRODIGE study recently noted that adjuvant gemcitabine and oxaliplatin (GEMOX) did not confer a survival benefit despite an acceptable safety/tolerance profile. ${ }^{34}$ The reasons for these disparate results are unclear, but may relate to the fact that there were more patients with $\mathrm{LN}$ metastasis and an $\mathrm{R} 1$ resection margin in the BILCAP study versus the PRODIGE trial. In turn, this may suggest that adjuvant therapy has a more beneficial effect among patients who have adverse prognostic factors (i.e. LN metastasis and R1 margins) relative to those individuals who do not.

\section{CONCLUSION}

The survival benefit derived from lymphadenectomy was poor among patients with major vascular invasion, CEA > $5.0 \mathrm{ng} / \mathrm{mL}$, and LNM in areas other than the hepatoduodenal ligament. Resection of three or more LNs was associated with the highest therapeutic value among patients with LNM.

DISCLOSURES Kota Sahara, Diamantis I. Tsilimigras, Katiuscha Merath, Fabio Bagante, Alfredo Guglielmi, Luca Aldrighetti, Matthew Weiss, Todd W. Bauer, Sorin Alexandrescu, George A. Poultsides, Shishir K. Maithel, Hugo P. Marques, Guillaume Martel, Carlo Pulitano, Feng Shen, Olivier Soubrane, B. Groot Koerkamp, Ryusei Matsuyama, Itaru Endo, and Timothy M. Pawlik have no conflicts of interest to declare.

\section{REFERENCES}

1. Chang KY, Chang JY, Yen Y. Increasing incidence of intrahepatic cholangiocarcinoma and its relationship to chronic viral hepatitis. J Natl Compr Cancer Netw. 2009;7(4):423-7.
2. Amini N, Ejaz A, Spolverato G, Kim Y, Herman JM, Pawlik TM. Temporal trends in liver-directed therapy of patients with intrahepatic cholangiocarcinoma in the United States: a populationbased analysis. J Surg Oncol. 2014;110(2):163-70.

3. Taylor-Robinson SD, Foster GR, Arora S, Hargreaves S, Thomas HC. Increase in primary liver cancer in the UK, 1979-94. The Lancet. 1997;350(9085):1142-3.

4. Shirabe K, Shimada M, Harimoto N, et al. Intrahepatic cholangiocarcinoma: Its mode of spreading and therapeutic modalities. Surgery. 2002;131(1 Suppl 1):S159-S164.

5. Shen WF, Zhong W, Xu F, et al. Clinicopathological and prognostic analysis of 429 patients with intrahepatic cholangiocarcinoma. World J Gastroenterol. 2009;15(47):5976-82.

6. Mavros MN, Economopoulos KP, Alexiou VG, Pawlik TM. Treatment and prognosis for patients with intrahepatic cholangiocarcinoma: systematic review and meta-analysis. JAMA Surg. 2014;149(6):565-74.

7. Kim DH, Choi DW, Choi SH, Heo JS, Kow AW. Is there a role for systematic hepatic pedicle lymphadenectomy in intrahepatic cholangiocarcinoma? A review of 17 years of experience in a tertiary institution. Surgery. 2015;157(4):666-75.

8. Weber SM, Jarnagin WR, Klimstra D, DeMatteo RP, Fong Y, Blumgart LH. Intrahepatic cholangiocarcinoma: resectability, recurrence pattern, and outcomes. $J$ Am Coll Surg. 2001;193(4):384-91.

9. Shimada K, Sano T, Nara S, et al. Therapeutic value of lymph node dissection during hepatectomy in patients with intrahepatic cholangiocellular carcinoma with negative lymph node involvement. Surgery. 2009;145(4):411-6.

10. de Jong MC, Nathan H, Sotiropoulos GC, et al. Intrahepatic cholangiocarcinoma: an international multi-institutional analysis of prognostic factors and lymph node assessment. J Clin Oncol. 2011;29(23):3140-5.

11. Endo I, Gonen M, Yopp AC, et al. Intrahepatic cholangiocarcinoma: rising frequency, improved survival, and determinants of outcome after resection. Ann Surg. 2008;248(1):84-96.

12. Luo X, Yuan L, Wang Y, Ge R, Sun Y, Wei G. Survival outcomes and prognostic factors of surgical therapy for all potentially resectable intrahepatic cholangiocarcinoma: a large single-center cohort study. $J$ Gastrointest Surg. 2014;18(3):562-72.

13. Amini N, Ejaz A, Spolverato G, Maithel SK, Kim Y, Pawlik TM. Management of lymph nodes during resection of hepatocellular carcinoma and intrahepatic cholangiocarcinoma: a systematic review. J Gastrointest Surg. 2014;18(12):2136-48.

14. Bagante F, Gani F, Spolverato G, et al. Intrahepatic cholangiocarcinoma: prognosis of patients who did not undergo lymphadenectomy. J Am Coll Surg. 2015;221(6):1031-1040. e1031-1034.

15. Jutric Z, Johnston WC, Hoen HM, et al. Impact of lymph node status in patients with intrahepatic cholangiocarcinoma treated by major hepatectomy: a review of the National Cancer Database. HPB. 2016;18(1):79-87.

16. Tokunaga MM, Ohyama $S$, Hiki $N$, Fukunaga $T$, Inoue $H$, Yamaka K, et al. Therapeutic value of lymph node dissection in advanced gastric cancer with macroscopic duodenum invasion: is the posterior pancreatic head lymph node dissection beneficial? Ann Surg Oncol. 2009;16(5):1241-6.

17. Sasako M, McCulloch P, Kinoshita T, Maruyama K. New method to evaluate the therapeutic value of lymph node dissection for gastric cancer. Br J Surg. 1995;82(3):346-51.

18. Kosuga T, Ichikawa D, Okamoto K, et al. Survival benefits from splenic hilar lymph node dissection by splenectomy in gastric cancer patients: relative comparison of the benefits in subgroups of patients. Gastric Cancer. 2011;14(2):172-7. 
19. Lin J-X, Huang C-M, Zheng C-H, et al. Is all advanced gastric cancer suitable for laparoscopy-assisted gastrectomy with extended lymphadenectomy? A case-control study using a propensity score method. Ann Surg Oncol. 2016;23(4):1252-60.

20. Ueno H, Mochizuki H, Hashiguchi Y, et al. Potential prognostic benefit of lateral pelvic node dissection for rectal cancer located below the peritoneal reflection. Ann Surg. 2007;245(1):80-7.

21. Aiko T, Sasako M. The new Japanese Classification of gastric carcinoma: points to be revised. Gastric Cancer. 1998;1(1):25-30.

22. Strasberg SM. Nomenclature of hepatic anatomy and resections: a review of the Brisbane 2000 system. J Hepatobiliary Pancreat Surg. 2005;12(5):351-5.

23. Amin MB, Greene FL, Edge SB, et al. The Eighth Edition AJCC Cancer Staging Manual: continuing to build a bridge from a population-based to a more "personalized" approach to cancer staging. CA Cancer J Clin. 2017;67(2):93-9.

24. Bhanji F, Topjian AA, Nadkarni VM, et al. Survival rates following pediatric in-hospital cardiac arrests during nights and weekends. JAMA Pediatr. 2017;171(1):39-45.

25. Clark CJ, Wood-Wentz CM, Reid-Lombardo KM, Kendrick ML, Huebner M, Que FG. Lymphadenectomy in the staging and treatment of intrahepatic cholangiocarcinoma: a population-based study using the National Cancer Institute SEER database. $H P B$. 2011;13(9):612-20.

26. Zhang XF, Chen Q, Kimbrough CW, et al. Lymphadenectomy for intrahepatic cholangiocarcinoma: has nodal evaluation been increasingly adopted by surgeons over time? A National Database analysis. J Gastrointest Surg. 2018;22(4):668-75.

27. Hu J, Chen F-Y, Zhou K-Q, et al. Intrahepatic cholangiocarcinoma patients without indications of lymph node metastasis not benefit from lymph node dissection. Oncotarget. 2017;8(69):113817-27.
28. Choi SB, Kim KS, Choi JY, et al. The prognosis and survival outcome of intrahepatic cholangiocarcinoma following surgical resection: association of lymph node metastasis and lymph node dissection with survival. Ann Surg Oncol. 2009;16(11):3048-56.

29. Yamashita Y, Taketomi A, Morita K, et al. The impact of surgical treatment and poor prognostic factors for patients with intrahepatic cholangiocarcinoma: retrospective analysis of 60 patients. Anticancer Res. 2008;28(4c):2353-9.

30. Morine Y, Shimada M, Utsunomiya T, et al. Clinical impact of lymph node dissection in surgery for peripheral-type intrahepatic cholangiocarcinoma. Surg Today. 2012;42(2):147-51.

31. Bagante F, Spolverato G, Weiss M, et al. Surgical management of intrahepatic cholangiocarcinoma in patients with cirrhosis: impact of lymphadenectomy on peri-operative outcomes. World $J$ Surg. 2018;42(8):2551-60.

32. Yamamoto M, Takasaki K, Yoshikawa T. Lymph node metastasis in intrahepatic cholangiocarcinoma. Jpn. J. Clin. Oncol. 1999;29(3):147-50.

33. Primrose JN, Fox RP, Palmer DH, et al. Capecitabine compared with observation in resected biliary tract cancer (BILCAP): a randomised, controlled, multicentre, phase 3 study. Lancet Oncol. 2019;20(5):663-73.

34. Edeline J, Benabdelghani M, Bertaut A, et al. Gemcitabine and oxaliplatin chemotherapy or surveillance in resected biliary tract cancer (PRODIGE 12-ACCORD 18-UNICANCER GI): a randomized phase III study. J Clin Oncol. 2019;37(8):658-67.

Publisher's Note Springer Nature remains neutral with regard to jurisdictional claims in published maps and institutional affiliations. 